\title{
PREVALENCIA DE Cryptosporidium EN TERNEROS EN EL VALLE DE UBATÉ - CHIQUINQUIRÁ (COLOMBIA)
}

\section{PREVALENCE OF Cryptosporidium IN CALVES IN THE UBATÉ - CHIQUINQUIRÁ VALLEY (COLOMBIA)}

\author{
Catalina Avendaño1, Joaquín Quílez², Caridad Sánchez-Acedo³
}

\begin{abstract}
${ }^{1}$ Microbióloga Agrícola y Veterinaria, Esp. Facultad de Medicina Veterinaria, Universidad de Ciencias Aplicadas y Ambientales U.D.C.A, Calle 222 No.55-37, Bogotá D.C., Colombia, cavendano@udca.edu.co ${ }^{2}$ Médico Veterinario, PhD. Universidad de Zaragoza, España, jquilez@unizar.es ${ }^{3}$ Médico Veterinario, PhD. Universidad de Zaragoza, España, csarmfm@unizar.es
\end{abstract}

Rev. U.D.C.A Act. E Div. Cient. 13 (1): 41-47, 2010

\section{RESUMEN}

Con el objeto de contribuir al conocimiento de la criptosporidiosis, reconocer la prevalencia de Cryptosporidium en terneros y asociar la presencia de ooquistes a la consistencia de la materia fecal, se realizó un estudio en el cual, se recolectaron 170 muestras de heces de terneros que tenían entre cinco y 35 días de edad, recogidas en 41 fincas ubicadas en el Valle de Ubaté - Chiquinquirá. Para identificar los ooquistes de Cryptosporidium, se empleó la técnica de Heine. El 22\% de las muestras fue positivo y en el $44 \%$ de las fincas evaluadas había, al menos, un animal infectado. El análisis estadístico mostró una asociación entre la edad y la presencia de ooquistes de Cryptosporidium ( $\mathrm{X}^{2} 15$; $\mathrm{P}<$ $0,01)$. La mayor prevalencia se halló en los terneros que tenían entre 11 - 15 y 16 - 20 días de nacidos, existiendo diferencias significativas al comparar estos dos grupos de edad frene a los demás grupos. Estadísticamente, no hubo una asociación entre la presencia o ausencia de Cryptosporidium y la consistencia de la materia fecal $\left(X^{2} 1,3 . P>0,25\right)$. Al comparar la consistencia de la materia fecal con la intensidad de la infección no se halló una asociación entre tales variables $\left(X^{2} 1,7\right.$. $\left.P>0,42\right)$, lo que pone de manifiesto que en el Valle de Ubaté Chiquinquirá existe un elevado número de terneros infectados asintomáticos. Los resultados obtenidos confirman que la infección por Cryptosporidium está ampliamente distribuida en las explotaciones de ganado vacuno, de la zona geográfica de estudio.
Palabras clave: Cryptosporidium, criptosporidiosis, terneros, heces, técnica de Heine.

\section{SUMMARY}

To contribute to the knowledge of cryptosporidiosis, recognize the prevalence of Cryptosporidium in calves and associate the presence of oocysts to the fecal consistence, a study was executed, collecting 170 samples of fecal material of calves, aged between five and 35 days, within 41 ranches located in the Ubaté-Chinquinquirá valley. In order to identify the Cryptosporidium oocysts the Heine technique was employed. Thirty seven (22\%) of the 170 calves were positive and 18 (44\%) of the 37 ranches showed at least one positive animal. The statistical analysis revealed an association between age and the presence of oocysts $\left(\mathrm{X}^{2} 15 ; \mathrm{P}<0.01\right)$. The highest prevalence was found in calves between age 11 to 15 and 16 to 20 days, presenting significant differences when comparing these two age groups with the other ones examined. Statistically, no association between the presence or absence of Cryptosporidium and the consistence of the fecal material was detected $\left(X^{2} 1.3\right.$; $P>0.25)$. When comparing the fecal consistence with the infection intensity, no association was found $\left(\mathrm{X}^{2}\right.$ 1.7; $\mathrm{P}>0.42$ ), result that confirms the high number of asymptomatic calves in the Ubaté-Chiquinquirá valley. The outcome of this research indicates an ample distribution of Cryptosporidium infection in the cattle ranches of the geographic area under survey. 
Key words: Cryptosporidium, cryptosporidiosis, calves, feces, Heine technique.

\section{INTRODUCCIÓN}

Cryptosporidium es un patógeno entérico común en terneros y otras especies de vertebrados (Schmidt $\varepsilon$ Kuhlenschmidt, 2008). La importancia de este parásito cada vez es más reconocida. En el 2004, la Organización Mundial de la Salud (OMS) lo identificó como un patógeno descuidado (Chalmers E Davies, 2010).

Cryptosporidium spp. pertenecen al phylum Apicomplexa (= Sporozoa), cuyos miembros poseen un complejo apical; clase Sporozoae, que se reproducen por ciclos sexuales y asexuales; subclase Coccidia, el ciclo de vida de ellos involucra merogonia, gametogonia y esporogonia; orden Euciccidiida (= Eucoccidiorida), en los que ocurre la esquizogonia; suborden Eimeriina (= Eimeriorina), en los cuales, la microgamia y al macrogamia, se desarrollan de manera independiente y familia Cryptosporidiidae, cuyos integrantes tienen cuatro esporozoitos desnudos dentro del ooquiste (Plutzer E Karanis, 2009).

La criptosporidiosis, se transmite mediante la ingestión de ooquistes esporulados, estadios infectantes que son eliminados en grandes cantidades en las heces de los animales y los humanos parasitados, durante la fase aguda de la infección y tienen una gran resistencia a las condiciones ambientales y desinfectantes habituales, manteniéndose infectantes durante periodos prolongados. Esta circunstancia, unida a la baja dosis infectante (10-100 ooquistes), el elevado número de especies animales que actúan como reservorios del parásito y la ausencia de un tratamiento farmacológico eficaz, facilitan la difusión de la enfermedad (Casemore et al. 1997).

El principal reservorio es el ganado bovino, específicamente, los terneros, en los que la criptosporidiosis es responsable de una alta tasa de morbilidad neonatal (Broglia et al. 2008). Cryptosporidium tiene un gran potencial de transmisión, a través del agua de bebida, debido a que los ooquistes pueden penetrar las barreras físicas usadas en el tratamiento de aguas, además de ser resistentes a los desinfectantes usados en este proceso, todo ligado a la baja dosis infectante para los humanos y los animales (Smith et al. 2007). La infección en los bovinos está altamente relacionada con la edad, las mayores prevalencias e intensidad de eliminación de ooquistes ocurren en los terneros jóvenes (Brook et al. 2008).

Las enfermedades diarreicas representan uno de los problemas fundamentales de salud en los terneros en el periodo neonatal. Cryptosporidium es un parásito reconocido como una de las principales causas de diarreas en terneros de hasta un mes de edad. Aunque la infección es responsable de una alta mortalidad, su impacto se asocia, principalmente, con el daño del intestino y la baja conversión del alimento. Este parásito causa tazas de morbilidad en las fincas, incluso el $100 \%$ en animales, de hasta un mes de edad (Klein et al. 2008). En las explotaciones ganaderas, la principal fuente de contagio la constituyen los propios animales enfermos, que infectan con sus heces la cama de la explotación. La prevalencia suele ser mayor en explotaciones con un elevado número de animales y determinados factores, como el hacinamiento y las condiciones higiénicas deficientes, que se consideran factores de riesgo. Las anteriores consideraciones justifican que la presentación clínica de la enfermedad esté asociada con la época de partos, observándose un marcado incremento en la incidencia de nuevos casos, al final de la misma, como consecuencia de la contaminación progresiva de la explotación a lo largo de la paridera. Los animales adultos también desempeñan un papel importante en la transmisión, puesto que pueden actuar como portadores asintomáticos que eliminan un reducido número de ooquistes, aunque suficiente para infectar a los animales recién nacidos (Vergara \& Quílez, 2004).

Un ternero con diarrea puede excretar $10^{7}$ ooquistes por gramo de heces y, así, puede producir billones de ooquistes, durante una a dos semanas donde la infección esta patente (O'Handley, 2007). Los tratamientos para la criptosporidiosis son más paleativos que curativos. El uso de calostro bovino hiperinmune ha demostrado una reducción de los síntomas clínicos de la enfermedad, así como de la reducción de la eliminación de ooquistes, en varias especies animales. De manera interesante, el calostro bovino no inmune también ha mostrado eficacia en personas inmunocomprometidas, lo cual, se puede deber a la presencia de algunos ácidos grasos insaturados (linoleico, oleico), que inhiben la adhesión de los esporozoitos al enterocito (Schmidt $\mathcal{E}$ Kuhlenschmidt, 2008). 
Por otra parte, la resistencia de los ooquistes a los tratamientos y a los desinfectantes utilizados rutinariamente para potabilizar el agua de bebida ha dado notoriedad a la criptosporidiosis, en los últimos años, como enfermedad de transmisión hídrica, que hoy en día, se considera como uno de los mecanismos de transmisión de la enfermedad al hombre, de mayor interés (Widme et al. 1996). De hecho, se han documentado un total de 39 brotes hídricos en el Reino Unido, Estados Unidos, Canadá y Japón (Slifko et al. 2000), destacando el ocurrido en la ciudad norteamericana de Milwaukee, en 1993, donde 400.000 personas resultaron afectadas, requiriendo ingreso hospitalario un $10 \%$ de ellos y, aproximadamente 100 murieron (Mac Kenzie et al. 1994).

La prevalencia de la criptosporidiosis en Sudamérica no se conoce con exactitud, puesto que los estudios epidemiológicos realizados son escasos, aunque el parásito ha sido identificado en todos los países en los que se investigó su presencia, mediante técnicas coprológicas (Vergara et al. 2001).

En Colombia, entre junio de 1996 y octubre de 1998, se realizó el primer estudio serológico de Cryptosporidium spp. en el cual se encontró una seroprevalencia de $83,3 \%$, indicando que la criptosporidiosis es endémica en el país y representa un importante problema de salud pública (Vergara et al. 2000).

Esta investigación buscó contribuir al conocimiento de la criptosporidiosis en Colombia, mediante un estudio epidemiológico en el Valle de Ubaté-Chiquinquirá, a través de la determinación de la prevalencia Cryptosporidium en terneros lactantes en explotaciones de ganado vacuno, así como la determinación de la edad de los terneros en que se produce la máxima receptividad a la infección y, adicionalmente, valorar la importancia de Cryptosporidium, como agente asociado con diarrea en terneros.

Los Valles de Ubaté - Chiquinquirá están conformados geográficamente porlas zonas relativamente bajas (2600msnm, en promedio), de los municipios de Ubaté, Susa, Fúquene, Lenguazaque, Simijaca, Guachetá, Cucunubá y Sutatausa del Departamento de Cundinamarca y Chiquinquirá, San Miguel de Sema, Ráquira y Saboya, del Departamento de Boyacá.
La zona presenta un amplio potencial competitivo para la producción de leche, tanto en el contexto nacional como en el internacional, ya que como señala el Estudio de Competitividad de la Cadena Láctea, citado por Valderama E Tellez (2003), tiene una provechosa ubicación geográfica, con buenas vías de acceso; excelente dotación de recursos naturales, topografía en su mayoría plana; tradición y cultura de la leche; aceptables niveles de calidad de leche; escala de producción competitiva y amplia presencia de empresas acopiadoras y transformadoras. Adicionalmente, la micro-cuenca ofrece una gran dinámica de crecimiento en la producción lechera y de participación en el mercado de Bogotá, ya que, como lo mencionan Valderrama E Téllez (2003), según el Centro de Estudios Agrícolas y Ganaderos CEGA, para 1990, se reportaban 238.080 litros diarios procedentes de la micro-cuenca y, en 1994, 517.973 litros, y de acuerdo a las entrevistas aplicadas en el 2001, se puede estimar que unos 582.000 litros diarios llegan a Bogotá, procedentes de la zona.

\section{MATERIALES Y MÉTODOS}

En el Valle de Ubaté - Chiquinquirá (Colombia), en un periodo de seis meses, se recolectaron 170 muestras de materia fecal de terneros lactantes entre cinco y 35 días de edad, pertenecientes a 41 explotaciones de ganado lechero. Los terneros fueron separados de la madre a los tres días, luego de haber ingerido calostro y, posteriormente, amarrados a estacas; la dieta suministrada en las fincas fue leche hasta los tres a cuatro meses de edad. Todas las explotaciones estudiadas, se dedicaban a la lechería.

Para alcanzar los objetivos del presente trabajo, se realizó un estudio transversal descriptivo, estimando el tamaño de la muestra, mediante el programa Win Episcope 2.0, sobre una población total esperada de 31.707 terneros, en el Valle de Ubaté - Chiquinquirá. Se consideró una prevalencia esperada del $50 \%$, cifra recomendada cuando se ignora la prevalencia aproximada (Thursfield, 2007). Tomando como condiciones básicas un intervalo de confianza del 95\% y un error del 7,5\%, el tamaño de la muestra ascendió a 170 terneros, los cuales, fueron seleccionados al azar entre el total de efectivos de las explotaciones existentes, en la zona geográfica de estudio.

Los ooquistes de Cryptosporidium, se identificaron mediante la tinción de Heine (Heine, 1982). La 
intensidad de la infección, se estimó empleando el promedio de ooquistes por campo microscópico de 100X, estableciéndose cuatro criterios de evaluación: Negativa, ausencia de ooquistes; leve, 0-6; moderada, $6-10$ y grave, $>10$ ooquistes).

Para analizar los resultados obtenidos, se elaboraron tablas cruzadas y se estimó la proporción; se calculó el intervalo de confianza del $95 \%$ y se realizaron asociaciones, a través del uso de la prueba de $\mathrm{X}^{2}$

\section{RESULTADOS Y DISCUSIÓN}

\section{Prevalencia de parasitación por Cryptosporidium en terneros lactantes}

Los ooquistes de Cryptosporidium fueron identificados en las heces de 37 terneros de 18 fincas (Figuras 1 y 2). Los resultados obtenidos confirman que la infección por Cryptosporidium está ampliamente distribuida en las explotaciones de ganado vacuno de la zona geográfica de estudio. En Colombia, solamente existe un estudio epidemiológico publicado sobre prevalencia de criptosporidiosis bovina (Vergara et al. 2001), que fue llevado a cabo mediante técnicas serológicos, lo cual, justifica que la prevalencia detectada por estos autores $(53,3 \%)$ fuera superior a la observada en el presente trabajo, ya que los niveles de anticuerpos séricos persisten durante varios meses, una vez que la infección ha remitido. Los datos obtenidos concuerdan con los hallazgos registrados por Surumay \& Alfaro (2000), en un trabajo realizado en Venezuela donde hallaron una prevalencia del 29,3\% en terneros; en Argentina, un estudio similar, reportó una prevalencia del 17\% (Del
Coco et al. 2008) cifra también cercana a la encontrada en este esta investigación.

\section{Edad de los terneros en que se produce la máxima receptividad a la infección}

La distribución de la edad de los animales infectados reveló que el mayor porcentaje de eliminación de ooquistes fue en los terneros que tenían entre 11 y 20 días de edad. El análisis estadístico mostró una asociación entre la edad y la presencia de ooquistes de Cryptosporidium $\left(X^{2} 15 P<0,01\right)$. Los terneros ubicados en el grupo de edades de 11-15 y 16-20 días de nacidos fueron los que tuvieron la mayor proporción de Cryptosporidium, existiendo diferencias significativas al comparar estos dos grupos de edad frente a los demás grupos. El rango de edad que manifiesta la mayor cantidad de terneros, con una intensidad leve, estuvo dado por los animales que tenían entre 16-20 días de edad y sólo en los terneros de 11-15 días, se observó alguno con una intensidad de parasitación grave.

El análisis estadístico mostró una asociación entre la edad y la presencia de ooquistes de Cryptosporidium $\left(X^{2} 15 P<0,01\right)$. Los terneros ubicados en el grupo de edades de 11-15 y 16-20 días de nacidos fueron los que tuvieron la mayor proporción de Cryptosporidium, existiendo diferencias significativas al confrontar estos dos grupos de edad contra los demás. Por otro lado, el $83 \%$ de los terneros tuvo presencia de ooquistes de Cryptosporidium mostrando una intensidad de infección leve, mientras que en el $14 \%$ fue moderada y solo en un $3 \%$ tuvo una infección grave.

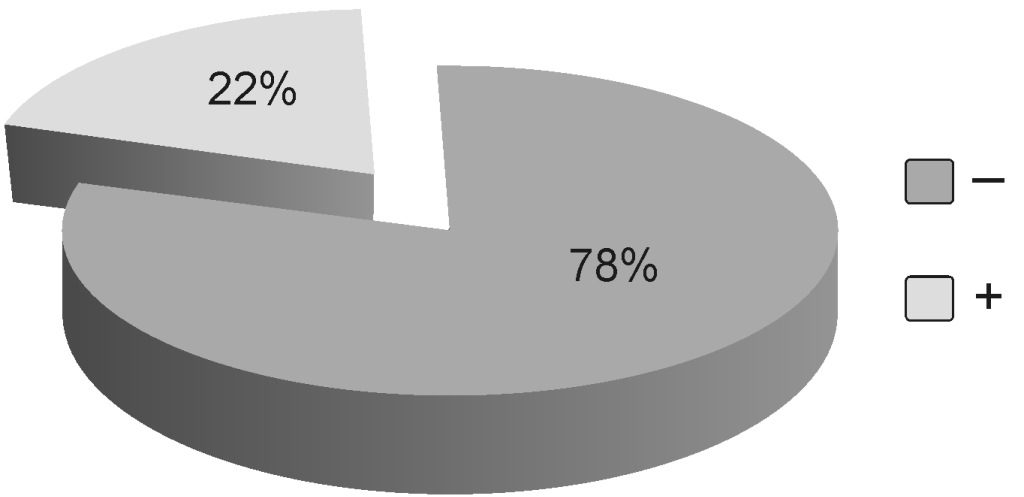

Figura 1. Proporción de muestras positivas. 


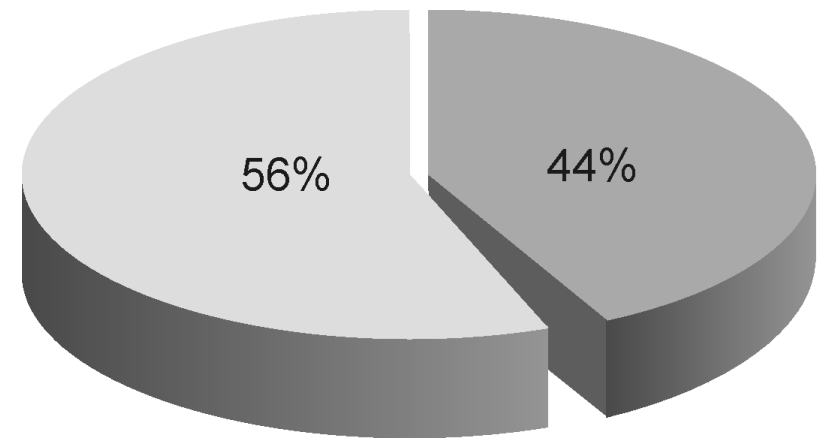

Fincas con al menos un animal positivo

Fincas sin animales positivos

Figura 2. Proporción de fincas positivas.

La mayor prevalencia de parasitación observada en terneros de 11 a 20 días de vida coincide con los resultados obtenidos por diversos investigadores, al señalar que la mayoría de los animales se infectan durante el periodo neonatal y la mayor receptividad a la infección, se manifiesta en las tres primeras semanas de vida de los rumiantes (Anderson, 1981; Ongerth $E$ Stibbs, 1989; Quílez et al. 1996).

\section{Importancia de Cryptosporidium, como agente asociado con diarrea en terneros}

Del total de terneros analizados, 78 padecían un síndrome diarreico en el momento de realizar la toma de muestras y 92 eran asintomáticos. La comparación con los resultados obtenidos en el estudio microscópico indicó que 20 (26\%) de los 78 terneros con diarrea estaban infectados por Cryptosporidium, mientras que el porcentaje de parasitación fue del 19\% (17/92), en los terneros que no padecían este síndrome. El análisis estadístico reveló que no existe una asociación estadísticamente significativa entre infección por Cryptosporidium y presencia o ausencia de diarrea, ni en el total de terneros analizados ni en cada uno de los grupos de edad por separado $(P>0,25)$.

En 51 de los terneros con diarrea, la consistencia de las heces fue clasificada como blanda y, en 13 de ellos, se detectaron ooquistes de Cryptosporidium (26\%). Los 27 terneros restantes padecían una diarrea líquida, donde siete (26\%), estaban parasitados. El estudio estadístico demostró que no existe una asociación estadísticamente significativa entre infección por Cryptosporidium y consistencia de las heces en terneros con diarrea $(P>1)$.
Al realizar el análisis estadístico comparando la consistencia de la materia fecal, según la intensidad de la infección, no se halló una asociación entre tales variables $\left(X^{2} 1,7 ; P>0,42\right)$.

Numerosos autores han señalado la importancia de Cryptosporidium como agente etiológico del síndrome de diarrea neonatal en terneros (Lassen et al. 2009; Joachim et al. 2003). En el presente trabajo, no se pudo demostrar la existencia de una asociación estadísticamente significativa, entre infección por este protozoo y presencia de diarrea, ni en el total de animales parasitados ni en cada uno de los grupos de edad por separado, a pesar que el $26 \%$ de los terneros afectados por el síndrome de diarrea neonatal estaban infectados por Cryptosporidium. Este hallazgo pone de manifiesto que en el valle de Ubaté y de Chiquinquirá existe un elevado número de terneros infectados asintomáticos, circunstancia ya descrita por otros autores (Lassen et al. 2009; Rings \& Rings, 1996), quienes afirman que la diarrea puede estar ausente aun en animales altamente infectados y los síntomas clínicos normalmente se presentan en la última fase del ciclo del parásito, cuando los quistes son eliminados en las heces.

Se encontró que la criptosporidiosis es una entidad que se manifiesta en la región colombiana bajo estudio, que tiene una prevalencia que se debe incluyir como entidad de importancia en los programas de salud bovina en el país y así avanzar en los estudios que permitan conocer más la epidemiología de la parasitosis.

Es preciso hacer hincapié que el estudio se realizó en trópico alto (frío); por la información conocida sobre el ciclo del parásito, se presume, entonces, que en el trópico 
cálido la prevalencia podría ser superior a la encontrada en el presente estudio. Esto obligará al grupo de estudio a adelantar investigaciones de mayor profundidad en ganaderías bovinas, tanto de lecheras como de producción de carne, en trópico cálido (bajo o cálido).

En Colombia, la morbi-mortalidad de terneros está bajo estudio y no se conocen las cifras del impacto verdadero de su mortalidad por síndromes diarreicos; sin embargo, se sabe que este componente es importante en ganaderías de alto nivel de manejo, donde se llevan registros y esa mortalidad allí es de más del $5 \%$ anual. $\mathrm{Si}$, se considera la valiosa importancia genética de esos individuos y el correspondiente alto valor económico, entonces, el impacto se presume costoso.

En el presente trabajo, se encontró el $22 \%$ de muestras positivas que oscilaron entre infestaciones leves (83\%), moderadas (14\%) y graves (3\%), lo que aconseja que será importante continuar los estudios en estas ganaderías, con el propósito de conocer en un año cómo se comporta la enfermedad.

Se reconoce que los resultados obtenidos en el trabajo corresponden a un momento en el tiempo y en el espacio y solamente haciendo un seguimiento seriado de los hatos, bajo estudio, será posible conocer la realidad epidemiológica de la criptosporidiosis, en esa área de trópico alto, en la cordillera oriental colombiana.

Sin embargo, de esta investigación se debe resaltar, que la infección por Cryptosporidium está ampliamente distribuida en las explotaciones de ganado vacuno del Valle de Ubaté y de Chiquinquirá y que la parasitación es significativamente mayor en terneros de dos a tres semanas de vida. Además, se destaca que, a pesar que Cryptosporidium fue identificado en un elevado porcentaje de los terneros con diarrea neonatal, no se observó una asociación estadística significante entre infección por este protozoo y la diarrea en terneros.

AGRADECIMIENTOS: Los autores agradecen al Doctor Ernesto González, Director del Comité de Ganaderos Zona 8, por su colaboración en la consecución de las muestras. Conflicto de intereses: Los autores del presente escrito declaran que no existe conflicto de intereses que ponga en riesgo la validez de los resultados.

\section{BIBLIOGRAFÍA}

1. ANDERSON, B.C. 1981. Patterns of shedding of cryptosporidial oocysts in Idaho calves. J. Am. Vet. Med. Assoc (Estados Unidos). 178(9):982-984.

2. BROGLIA, A.; RECKINGER, S.; CACCIÓ, S.; NÖCKLER, K. 2008. Distribution of Cryptosporidium parvum subtypes in calves in Germany. Vet. Parasitol. (Paises Bajos). 154(1-2):8-13.

3. BROOK, E.; HART, C.; FRENCH, N.; CHRISTLEY, R. 2008. Prevalence and risk factors for Cryptosporidium spp. infection in young calves. Vet. Parasitol. 152(1-2):46-52.

4. CASEMORE, D.; WRIGHT, S.; COOP, R. 1997. Cryptosporidiosis - human and animal epidemiology. En: Fayer, R. ed. Cryptosporidium and cryptosporidiosis. CRC Press, Boca Ratón, Florida. p.65-92.

5. CHALMERS, R.; DAVIES, A. 2010. Minireview: Clinical cryptosporidiosis. Experimental Parasitol. (Estados Unidos). 124(1):138-146.

6. DEL COCO, V.F.; CÓRDOBA, M.A.; BASUALDO, J.A. 2008. Cryptosporidium infection in calves from a rural area of Buenos Aires, Argentina. Vet. Parasitol. 158(1-2):31-35.

7. JOACHIM, A.; KRULL, T.; SCHWARZKOPF, J.; DAUGSCHIES, A. 2003. Prevalence and control of bovine criptosporidiosis in german dairy herds. Vet. Parasitol. 112(4):277-288.

8. HEINE, J. 1982. A simple technic for the demonstration of cryptosporidia in feces. Zentralbl. Veterinarmed. B. (Alemania). 29(4):324-327.

9. KLEIN, P.; KLEINOVÁ, T.; VOLEK, Z.; ŠIMŮINEK, J. 2008. Effect of Cryptosporidium parvum infection on the absorptive capacity and paracellular permeability of the small intestine in neonatal calves. Vet. Parasitol. 152(1-2):53-59.

10. LASSEN, B.; VILTROP, A.; RAAPERI, K.; JÄRVIS, T. 2009. Eimeria and Cryptosporidium in Estonian dairy farms in regard to age, species, and diarrhea. Vet. Parasitol. 166(3-4):212-219. 
11. MAC KENZIE, W.; HOXIE, N.; PROCTOR, M.; GRADUS, M.; BLAIR, K.; PETERSON, D.; KAZMIERCZAK, J.; ADDISS, D.; FOX, K.; ROSE, J. 1994. A massive outbreak in Milwaukee of Cryptosporidium infection transmitted through the public water. N. Engl. J. Med. (Estados Unidos). 331(3):161-167.

12. O'HANDLEY, R. 2007. Cryptosporidium parvum infection in cattle: are current perceptions accurate? Trends Parasitol. (Inglaterra). 23(10):477-480.

13. ONGERTH, J.; STIBBS, H. 1989. Prevalence of Cryptosporidium infection in dairy calves in western Washington. Am. J. Vet. Res. (Estados Unidos). 50(7):1069-1070.

14. PLUTZER, J.; KARANIS, P. 2009. Genetic polymorphism in Cryptosporidium species: an update. Vet. Parasitol. 165(3-4):187-199.

15. QUÍLEZ, J.; SÁNCHEZ-ACEDO, C.; DEL CACHO, E.; CLAVEL, A.; CAUSAPÉ, A. 1996. Prevalence of Cryptosporidium and Giardia infections in cattle in Aragón (northeastern Spain). Vet. Parasitol. 66(3-4):139-146.

16. RINGS, D.; RINGS, M. 1996. Managing Cryptosporidium and Giardia infections in domestic ruminants. Vet Med. (Estados Unidos). 91(12):11251130.

17. SCHMIDT, J.; KUHLENSCHMIDT, M. 2008. Microbial adhesion of Cryptosporidium paruum: Identification of a colostrum-derived inhibitory lipid. Mol. \& Biochem. Parasit. (Paises Bajos). 162(1):32-39.

18. SLIFKO, T.; SMITH, H.; ROSE, J. 2000. Emerging parasite zoonoses associated with water and food. Int. J. Parasitol. (Inglaterra). 30(12-13): 13791393.
19. SMITH, H.; CACCIÒ, S.; COOK, N.; NICHOLS, R.; TAIT, A. 2007. Cryptosporidium and Giardia as foodborne zoonoses. Vet. Parasitol. 149(1-2):2940.

20. SURUMAY, Q.; ALFARO, C. 2000. Cryptosporidium spp. in farms in the eastern region of Venezuela. Invest Clin. (Venezuela). 41(4):245-250.

21. THRUSFIELD, M. 2007. Veterinary Epidemiology. Third ed. Blackwell Publishing. (Oxford). p232 234.

22. VALDERRAMA, P.; TÉLLEZ, G. 2003. Microcuenca lechera Valles de Ubaté y Chiquinquirá. Caracterización y mercadeo de la leche. U. N. de Colombia, Facultad de Medicina Veterinaria y Zootecnia. http://www.veterinaria.unal.edu.co/inv/gigep/ Microcuenca\%20Lechera\%20_Libro_.pdf. (con acceso 28/12/09).

23. VERGARA, C.; QUÍLEZ, J. 2004. Criptosporidiosis: una zoonosis parasitaria. MVZ-Córdoba. (Colombia). 9:(1):363-372

24. VERGARA, C.; QUÍLEZ, J.; FREIRE, J.; CASTRO, J.; ARES, M. 2001. Serological response to Cryptosporidium paruum in adult cattle from the Andean region of Colombia. Parasitol. Res. (Alemania). 87(6):500-504.

25. VERGARA, C.; SANTOS, S.; FREIRE, F.; ARES, E. 2000. La criptosporidiosis en la región andina de Colombia: seroprevalencia y reconocimiento de antígenos. Rev. Panam. Salud Pública. (Estados Unidos). 8(6):373-379.

26. WIDME, G.; CARRAWAY, M.; TZIPORI, S. 1996. Water-borne Cryptosporidium: A perspective from the USA. Parasitol. Today. (Inglaterra). 12(7):286290.

Recibido: Diciembre 4 de 2009

Aceptado: Marzo 20 de 2010 